\title{
GROUND PENETRATING SYNTHETIC PULSE RADAR PERFORMANCES
}

\author{
J. Cariou, O. Blondel, E. Ferrari \\ Centre d'Etudes Techniques de l'Equipement \\ 76121 Grand Quevilly France
}

Four years ago, the «Ministère de l'Equipement et des Transports», in collaboration with two other French companies (SNCF - Société Nationale des Chemins de Fer; and the RATP Régie Autonome des Transports Parisiens) presented a paper concerning the development of a synthetic pulse radar. After demonstrating the feasibility of such a system at the end of the eighties, we have recently improved our knowledge and carried out comparisons with the pulse and synthetic radars existing on the market. The main purpose of this research is to try to improve the depth of investigation for the detection of discontinuity or cavities in the ground or in civil engineering structures.

Remember that synthetic pulse radars acquire information in the frequency domain. For each frequency analysed, the system records the characteristics (amplitude and phase shift) in transmission or reflection mode by the structure. Display of the echoes in the time domain is obtained by reverse Fourier transformation. The measurements in transmission correspond to bistatic operation, and the measurements in reflection to monostatic mode. As regards the results presented, we are limited to the work carried out in the frequency band ranging from 200 to $1200 \mathrm{MHz}$. The comparison will be made with a pulse radar associated to antennas of central frequency $500 \mathrm{MHz}$.

\section{PERFORMANCE ANALYSIS CRITERIA}

To compare the various radar solutions, we have adopted a physical criterion called Dynamic (performance Ratio) in $\mathrm{dB}$. It is defined as being the maximum attenuation that the electronic wave can undergo to be received. This dynamic is clearly not independent from the signal processing, and we will therefore specify the cases where processing has been carried out. The main part of our comparisons will not take into account the weight of this mathematical processing.

\section{THEORETICAL DYNAMIC}

The theoretical limit of the dynamic of a network analyser is often presented in the frequency domain as being linked to the thermal noise of the system. It turns out in fact, that the quality of the measurement generally prevents this limit from being reached. The phase and amplitude measurements carried out in the frequency domain are always affected by errors, making it difficult to reach the level of thermal noise. These two errors are mainly linked:

- to the characteristics of the equipment,

- to the operating conditions. 
Moreover, displaying information in the time domain involves Fourier transformations and several related calculations (filtering) which may introduce secondary lobes on the signal.

The maximum indicated (without processing the signal) is therefore difficult to reach under actual operating conditions in situ. Amongst the main causes of reduction in performance in the time domain, we may mention:

- The level of the received signal,

- The connection mode between the network analyser and the antennas,

- The auscultation speed,

- The antennas,

- The reflections linked to the environment.

During reconstruction of the time signal, we have noticed that the errors on the amplitude and phase measurements increased with the signal level. The following table illustrates this relationship between the noise level and the signal for two network analysers. This demonstrates the benefit of working in bistatic mode to avoid surface reflections whose level is likely to reduce the dynamic.

Signal amplitude Noise level of network analyser 1 Noise level of network analyser 2

$\begin{array}{rrr}-10 \mathrm{~dB} & -80 \mathrm{~dB} & -85 \mathrm{~dB} \\ -30 \mathrm{~dB} & -102 \mathrm{~dB} & -98 \mathrm{~dB} \\ -50 \mathrm{~dB} & -122 \mathrm{~dB} & -98 \mathrm{~dB} \\ \text { null } & -125 \mathrm{~dB} & -122 \mathrm{~dB}\end{array}$

These results were obtained with simple calibration and a $10 \mathrm{~m}$ connection cable and we noticed that the presence of this cable increased the noise level by $10 \mathrm{~dB}$ for network analyser 2. This deterioration does not exist in the other case because of signal transfer at low frequency.

This dynamic also depends on the width of the filter used to measure the amplitude and phase of each frequency. We found that a dynamic of $100 \mathrm{~dB}$ required a filter width of about 1 $\mathrm{kHz}$. In this case, the time required for auscultation of all frequencies was between $0.2 \mathrm{~ms}$ and $0.5 \mathrm{~ms}$. This indicates the maximum rate that the current network analysers can achieve. A further gain of $10 \mathrm{~dB}$ on the dynamic leads to an increase by a factor of 10 in the auscultation times which are therefore closely linked to the width of these filters.

After studying various solutions, we have defined by laboratory simulation operating conditions offering a theoretical dynamic of $110 \mathrm{~dB}$ in the time domain. This result takes into account neither the influence of the antennas nor that of the environment. The response in bistatic mode of a cavity buried in the ground was in this case simulated by a 3 meter connection cable followed by a $50 \mathrm{~dB}$ attenuator.

\section{REAL DYNAMIC}

A series of comparisons was carried out on an experimental site containing a cavity buried in clayey material. The echo level for this cavity was $-70 \mathrm{~dB}$ for the echo entering the cavity and $-80 \mathrm{~dB}$ for the echo from the lower surface of the cavity. We tested successively:

- a pulse radar No. 1

- a synthetic radar No. 2

- our experimental radar No. 3 
The first two are tools currently available on the market. Initially they were incapable of detecting these echoes, with or without single processing. The dynamic of these two radars is therefore around $60 \mathrm{~dB}$. Radar No. 2 however was able to show these echoes with improved antennas and with suitable signal processing. On the other hand, detection of these echoes is easily carried out with our assembly, and without signal processing.

\section{CONCLUSION}

We have demonstrated that radar dynamic can be improved by $50 \%$ by using a synthetic pulse radar, leading to an increase in the depth of action of the same proportion. The details of this development will be discussed during the congress. The only disadvantage with this solution lies in the limitation of auscultation speed. With current techniques, it is impossible to exceed a speed of 5 auscultations per second. Certain improvements will allow this performance to be doubled. 
